\title{
Metastatic differentiated thyroid cancer with undetectable serum thyroglobulin: diagnostic, management and follow-up challenges
}

Pedro Marques ${ }^{1}$, Teresa C. Ferreira ${ }^{2}$, Lucília Salgado ${ }^{2}$, Rafael Cabrera ${ }^{3}$, Valeriano Leite ${ }^{1}$ ${ }^{1}$ Endocrinology Department, Portuguese Institute of Oncology, Lisbon

2Nuclear Medicine Department, Portuguese Institute of Oncology, Lisbon

${ }^{3}$ Pathology Department, Portuguese Institute of Oncology, Lisbon

\section{Introduction}

- Serum thyroglobulin $(\mathrm{Tg})$ is a reliable marker in patients with differentiated thyroid carcinoma (DTC). Distant metastases of DTC usually presents with higher levels of $\mathrm{Tg}$, which are useful to follow the disease evolution'.

- Tg levels depend on tumor type, volume and proportion of cells synthesizing $\mathrm{Tg}$ within the tumor. Molecular heterogeneity of $\mathrm{Tg}$ due to diverse mRNA alternative splicing, heterogeneity in glycosylation and iodination, and the use of different $\mathrm{Tg}$ assays may also explain variable concentrations of $\mathrm{Tg}^{2}$.

\section{Case report}

- A 52-year-old woman underwent a subtotal thyroidectomy in 1993 because of a growing nodule. The histology was follicular thyroid carcinoma

- Eight years later, multiple lung nodules were identified in a thorax-CT scan. A biopsy of one of the pulmonary lesions was compatible with metastatic thyroid carcinoma (Figure 1). She was referred to our hospital.

- The thyroidectomy was completed and seven radioiodine treatments were administered (total activity of $800 \mathrm{mCi}$ ). The tail-end-scan following each treatment showed uptake in the lung metastases (Figure 2), except of the last scan where lung uptake was lost. Stimulated-Tg throughout these radioiodine treatments was consistently undetectable $(<0.2 \mathrm{ng} / \mathrm{mL})$ with TSH between 40 $93 \mathrm{mIU} / \mathrm{mL}$.

- During the 13-years follow-up, suppressed-Tg has been consistently undetectable and the measurement of $\mathrm{TgAb}$ negative, despite the progression in size and number of metastatic lesions (Figure 3). The non-radioiodine avid lung metastasis were proven to be fluorodeoxyglucose (FDG)-avid (Figure 2).

- Currently, the patient is asymptomatic with a progressive metastatic DTC, without biochemical evidence of the disease - undetectable $\mathrm{Tg}$ and $\mathrm{TgAb}$ (tested by 3 different laboratorial assays).

\section{Discussion}

$>$ Serum $\mathrm{Tg}$ is an excellent tumor marker for DTC, and serial monitoring is valuable for the follow-up of this condition. DTC with systemic dissemination usually has measurable $\mathrm{Tg}$.

$>$ Undetectable serum Tg in recurrent/metastatic DTC may not necessarily predict neither radioiodine uptake nor adverse prognosis, but it imposes important diagnostic, management and follow-up challenges. In such uncommon cases, imaging methods (CT, MRI and PET) should supplement $\mathrm{Tg}$ and $\mathrm{TgAb}$ measurements in order to allow a correct follow-up and management strategy.

Westburey C, Vini L, Fisher C, Harmer C. Recurrent differentiated thyroid cancer without elevation of serum thyroglobulin. Thyroid 2000:10(2):171-176 2. Giovanella $L$, Clark $P$, Chiovato $L$, Duntas $L$, Elisei $R$, et al. Thyroglobulin measurement using highly sensitive assays in patients with differentiated thyroid cancer: a clinical position paper. Eur J Endocrinol 2014;171:R33-R46
E-mail: pedro.miguel.sousa.marques@gmail.com

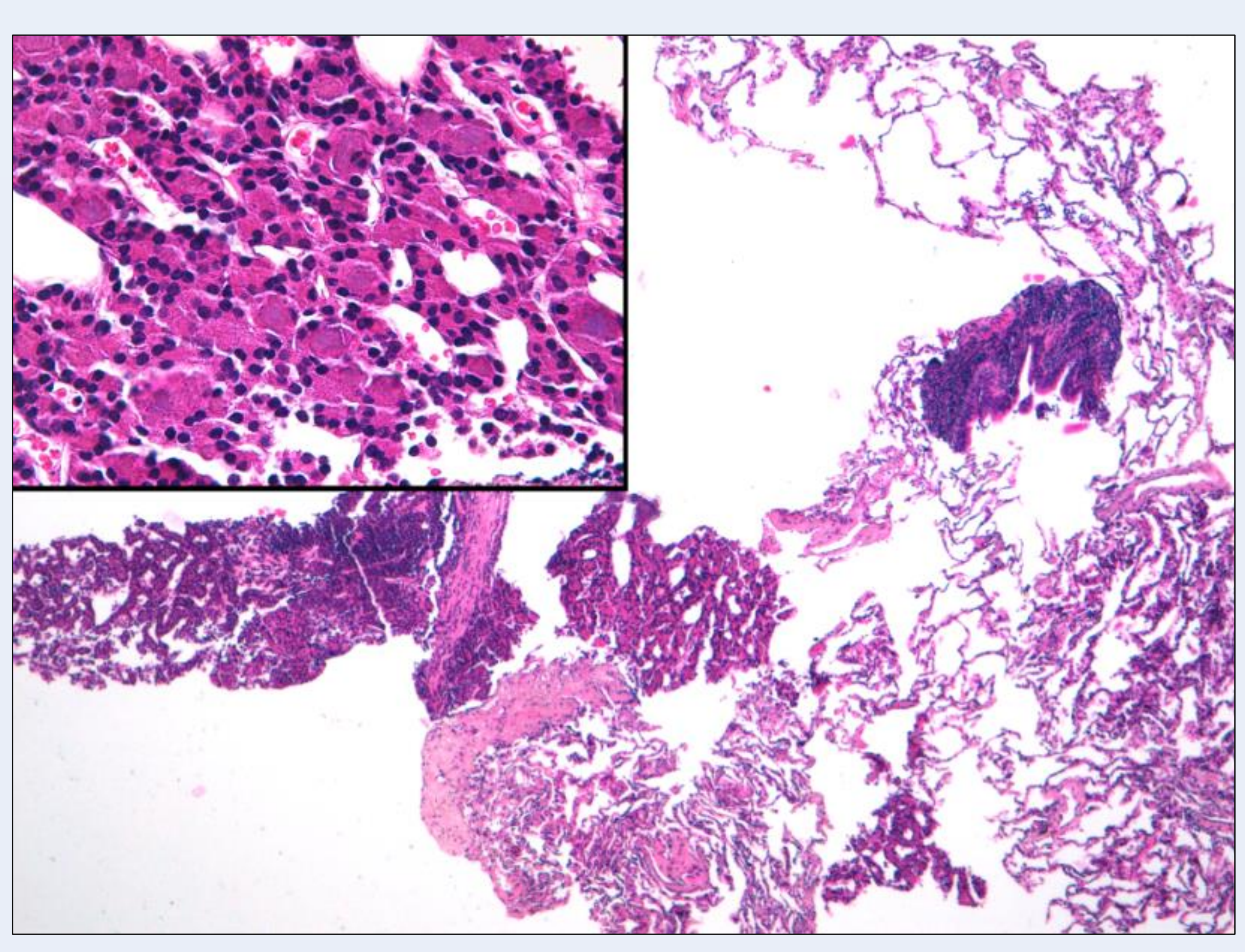

Figure 1: Histological analysis of the lung showing metastatic well-differentiated thyroid carcinoma (hematoxylin-eosin stain)

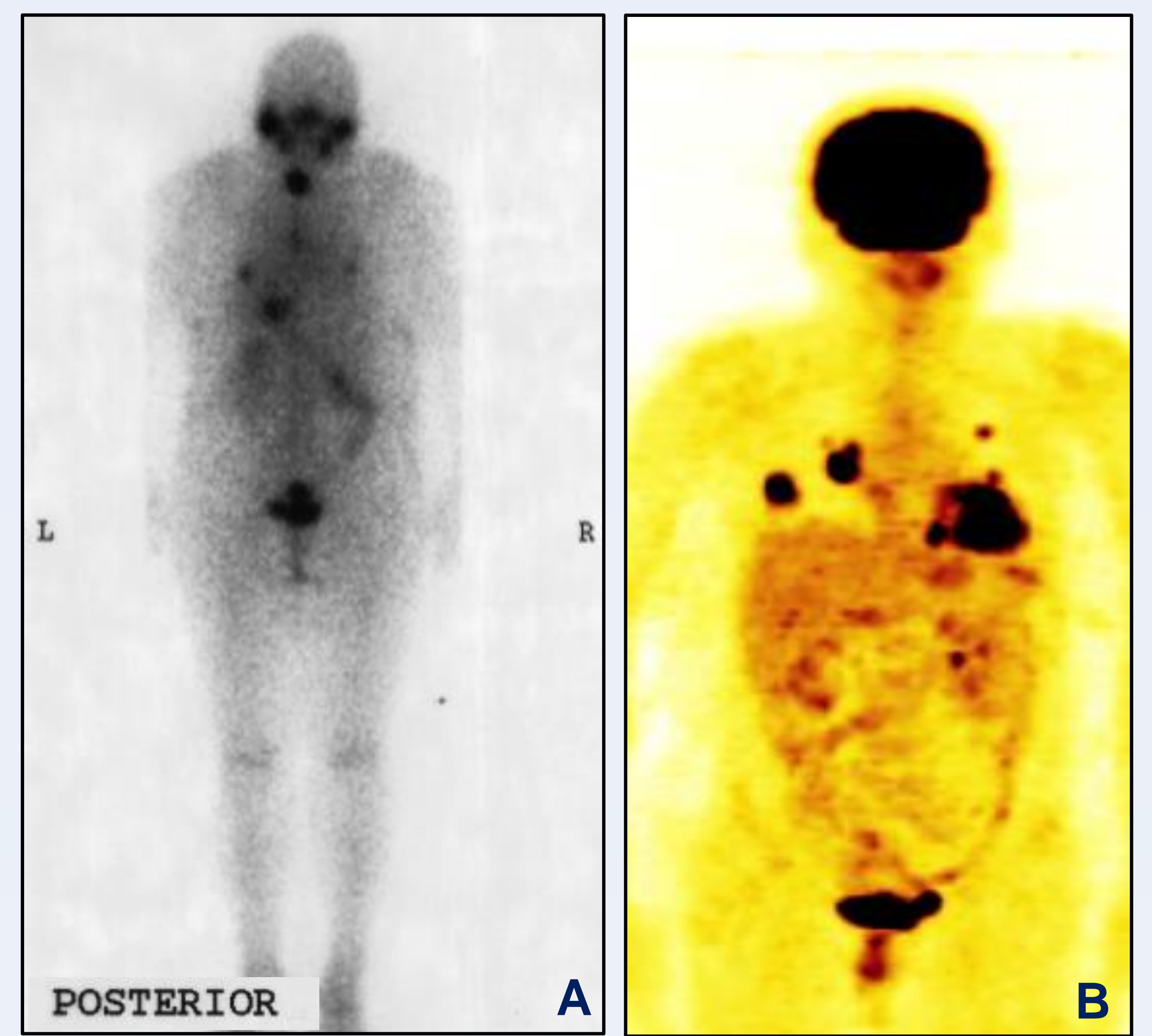

Figure 2: Post-radioiodine whole-body scan showing iodine-avid lung lesions (A); FDG-PET scan showing uptake in the lung metastasis (B).

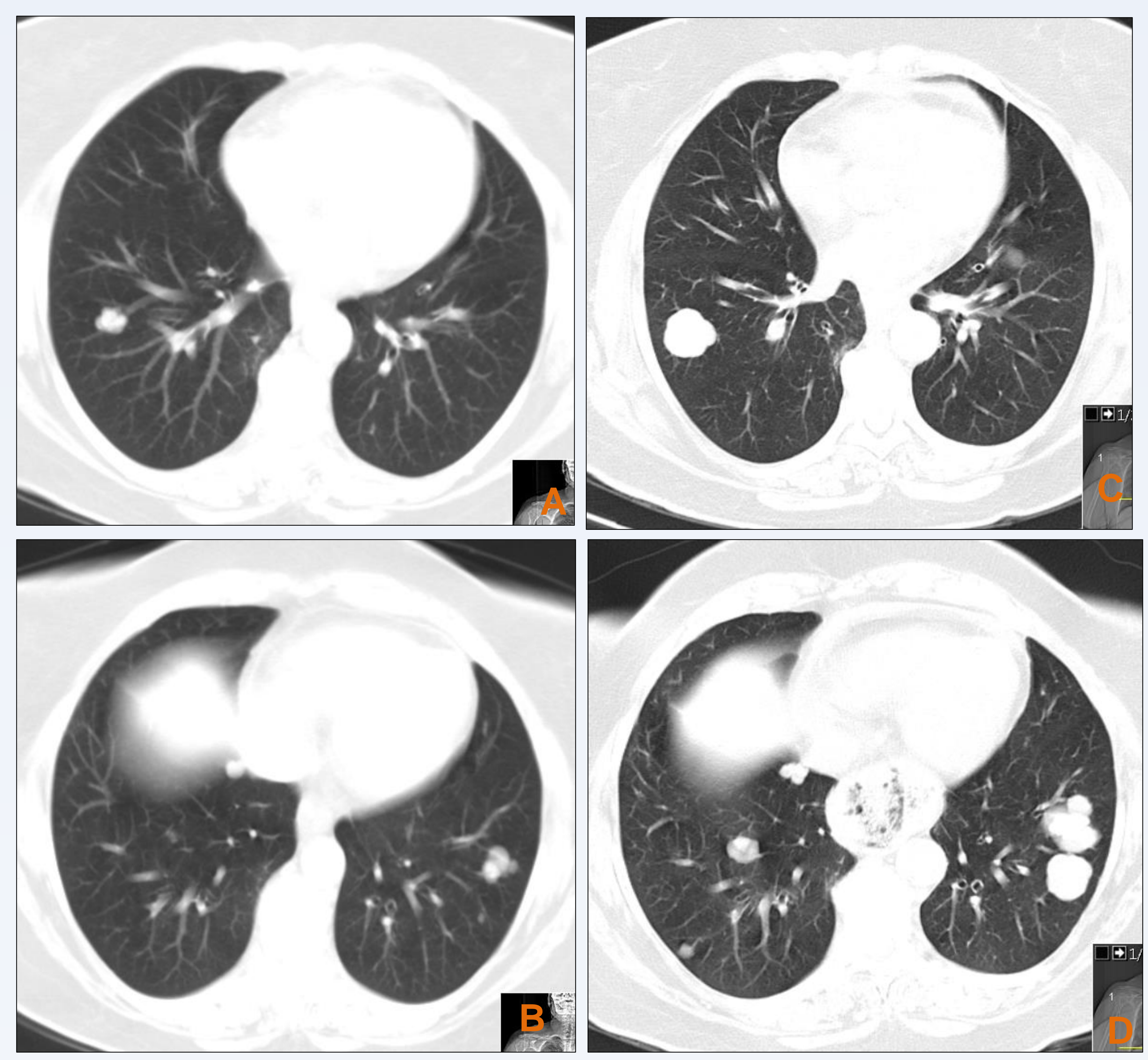

Figure 3: CT evaluation of the lung secondary lesions, at diagnosis $(A, B)$ and 13-years after (C,D). 